\title{
Intestinal preparation for elective laparoscopic colorectal surgery in elderly patients
}

\author{
E Mion ${ }^{1 *}$, F Viano $^{1}$, C Terranova ${ }^{2}$, S Ricciardi ${ }^{1}$, R Gianesini ${ }^{1}$, S Faccin ${ }^{1}$, L De Santis ${ }^{1}$ \\ From de Senectute: Age and Health Forum \\ Catanzaro, Italy. 5-7 December 2009
}

\section{Background}

The aim of the study was to compare the outcome of two groups of patients undergoing elective laparoscopic colorectal surgery, the first group receiving mechanical bowel preparation before elective colorectal surgery the second with no preparation.

\section{Materials and methods}

In our division, since 2002 elective colon surgery is routinely performed by laparoscopy.

Until September 2008 no preparation was done; from September 2008 a preparation based on polyethylene glycol was administered to all patients.

The study included 61 patients, aged above 60 who had undergone elective laparoscopic colorectal surgery. They were subdivided into two groups:

1. Group 1. N.33 patients average age 65 (between 60 and 89) were prepared before surgery; 7 patients underwent right hemicolectomy, 16 left hemicolectomy-sigmoidectomy and 10 anterior resection of the rectum.

2. Group 2. N.28 patients average age 64 (between 60 and 87) were not prepared before surgery; 7 patients underwent right hemicolectomy, 13 left hemicolectomy-sigmoidectomy and 8 anterior resection of the rectum.

\section{Results}

Group 1. Complications were described in 2 cases: one case of anastomotic dehiscence after left hemicolectomy resolved with conservative treatment; one case of anastomotic dehiscence in anterior resection of the rectum treated by laparoscopic drainage and ileostomy.

'Division of Surgery, Ospedale "San Lorenzo", Valdagno, Italy
Group 2. Complications were described in 7 cases: 2 cases of surgical wound infection, 4 cases of anastomotic fistulas two treated with conservative treatment, two treated with laparoscopic drainage and ileostomy, one case of abdominal sepsis, which caused the death of the patient.

The differences between the two groups in the number of complications were not statistically significant but still show a trend toward fewer complications in Group 1.

\section{Conclusions}

In our division, after the experience of colorectal surgery without bowel preparation it was decided to prepare patients for the following reasons:

1) in non-prepared patient an anastomotic fistula tends to be a more serious complication due to the fecal leakage in the abdominal cavity;

2) interventions in laparoscopy imply performing mechanical anastomoses that are easily carried out in prepared patients;

3) in our division the protective ileostomy is not performed routinely; in cases of anastomotic dehiscence in an unprepared patient you have a high risk of stercoraceous peritonitis.

\section{Author details}

'Division of Surgery, Ospedale "San Lorenzo", Valdagno, Italy. ${ }^{2}$ Department of Environmental medicine and public health, Hospital-University of Padova, Italy.

Published: 19 May 2010

doi:10.1186/1471-2318-10-S1-A15

Cite this article as: Mion et al:: Intestinal preparation for elective

laparoscopic colorectal surgery in elderly patients. BMC Geriatrics 2010 10(Suppl 1):A15. 